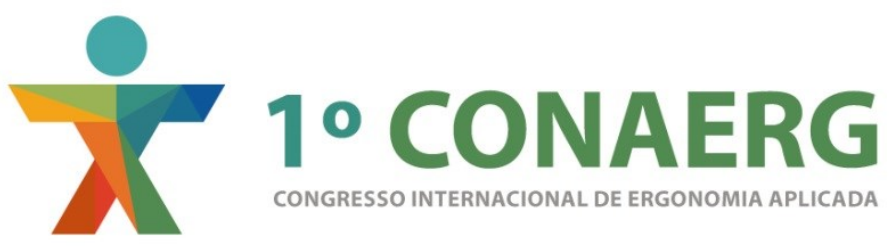

\title{
ERGONOMIA E PROMOÇÃO DE SAÚDE NO AMBIENTE DE TRABALHO: RELATO DE EXPERIÊNCIA DA INTERVENÇÃO EM TERAPIA OCUPACIONAL
}

\author{
Ana Karina Pessoa da Silva Cabral (1) \\ Danielle Carneiro de Menezes Sanguinetti (2) \\ Camila Beatriz Inácio Rodrigues dos Santos (3) \\ Marília Lopes Fonseca (3) \\ Raquel de Lima França (3) \\ Yury Dynnallyson Ferreira Oliveira (3) \\ (1) Universidade Federal de Pernambuco, Doutoranda em Design pela UFPE \\ e-mail: anakarinapessoa@yahoo.com.br \\ (2) Universidade Federal de Pernambuco, Doutora em Neuropsiquiatria e Ciências do \\ Comportamento, na área de Neurociências, pela UFPE \\ e-mail: dcmsanguinetti@gmail.com
}

(3) Universidade Federal de Pernambuco, Graduandos em Terapia Ocupacional pela UFPE

e-mail: pe.yury.dyn@gmail.com

\begin{abstract}
RESUMO
Ao processo de trabalho atribuiu-se uma gama de recursos tecnológicos ao longo do tempo, gerando especificidade à função e ônus à saúde. Este estudo objetivou apresentar estratégias de intervenções da Terapia Ocupacional no ambiente de trabalho como recurso de promoção de saúde, sob a ótica da Ergonomia. Trata-se de um relato de experiência, cujos dados foram coletados utilizando o Método de Análise Ergonômica do Trabalho. Realizaram-se grupos focais com as equipes de servidores de limpeza de uma instituição. Concluiu-se que programas de promoção a Saúde do Trabalhador surgem como importantes estratégias de reforço a saúde laboral, segundo a perspectiva analisada.
\end{abstract}

Palavras-chave: Ergonomia, Saúde do Trabalhador, Qualidade de vida.

\begin{abstract}
The work process has been assigned a range of technological resources over time, generating a specificity function and health burden. About this context changes arise in the sense of health at work and the prevention of diseases and accidents. This study presents strategies of health promotion and workplace interventions. This is a report of experience in practical classes. The data were collected through interview and ergonomic analysis of the Work of teams of cleaning servers and provided the basis for focus groups. The health promotion programmes of the worker appear as important strategies to strengthen the health, according to the analyzed perspective.
\end{abstract}

Keywords: Human Engineering, Occupational Health, Quality of Life. 


\section{INTRODUÇÃO}

O homem ao transformar o espaço ao seu redor reforça seus papeis como indivíduo social e humano. Ao realizar uma tarefa é reconhecido por ela e com isso se define no papel atribuído a mesma. A dinâmica envolvida no processo de manutenção de papéis gera relações sociais, o que propicia a criação de identidade humana através de uma atividade. A esse processo dá-se o nome de trabalho e a ele é atribuído a identidade e papéis humanos (KANTORSKI, 1997).

O trabalho assume um papel de grande significado na vida dos seres humanos, uma vez que na idade adulta se apresenta como a principal ocupação a ser realizada, requerendo dedicação de esforço e tempo dentro das organizações. Como meio de se manter, o trabalho também é um elemento importante, mas além do sentido financeiro, esse espaço contribui na constituição da identidade individual e possui implicação direta nas diversas formas de inserção social e no desempenho de atividades dos indivíduos (JUNQUEIRA, 2008; LANCMAN, 2011).

Desde as primeiras sociedades humanas até os dias atuais, o trabalho assumiu diversos significados. Antes servindo para a manutenção e a reprodução biológica do ser humano, se desempenhou sob a forma de coleta, de trabalho extrativo e mais tarde com a pesca, a caça e o pastoreio. Na agricultura, veio a ideia de que se produzindo um excedente do que se poderia utilizar, seria possível escravizar pessoas. Com a Revolução Industrial, que se iniciou no século XVIII, as fábricas juntaram num só espaço trabalhadores e os novos meios de produção, as máquinas, aumentando a especialização das tarefas e a divisão do trabalho, contribuindo para o afastamento das pessoas do conteúdo de suas próprias atividades, estimulando a longa jornada, a obsessão pela carreira e interferindo na saúde e bem-estar dos trabalhadores (MORETTI, TREICHEL, 2003).

Serra et al. (2015) afirmam que se por um lado as novas tecnologias trouxeram inovações de produtos e ferramentas para o mercado e linhas de produção, por outro lado essa nova forma de realizar tarefas e de manusear equipamentos cada vez mais complexos, permitiu o favorecimento de doenças relacionadas a rotina de trabalho.

$\mathrm{Na}$ década de 70 , devido à preocupação com a competitividade internacional e o grande sucesso dos estilos e técnicas gerenciais dos programas de produtividade japonesa, centrado nos empregados, surge um movimento pela qualidade de vida no trabalho. De acordo com Rodrigues (1994, p.76) apud Moretti e Treichel (2003):

"A qualidade de vida no trabalho tem sido uma preocupação do homem desde o início de sua existência com outros títulos em outros contextos, mas sempre voltada para facilitar ou trazer satisfação e bem-estar ao trabalhador na execução de sua tarefa".

A manutenção de postura e repetição de movimentos são fatores facilmente identificados nos processos de trabalho atuais, esses podem relacionar-se também com o surgimento de dor e desconforto corporal. De forma que, segundo De Paula (p.126, 2016) "a realização de qualquer trabalho exige, por parte do trabalhador, participação do corpo inteiro, o que pode sobrecarrega-lo ao longo da jornada de trabalho".

De acordo com Da Silva et al. (2006), sob essa perspectiva, vêm sendo implantadas políticas e ações na área de Saúde do Trabalhador a fim de promover a saúde no ambiente de trabalho, reduzindo acidentes e exposição a riscos de trabalho, sendo, portanto, fatores observados na casualidade dos afastamentos, juntamente com as doenças ocupacionais, estas que surgem com o reforço excessivo de sobrecarga de trabalho, dor e desconforto corporal no ambiente de trabalho.

De Paula (2016) afirma que a Ergonomia é compreendida como o estudo relacional do planejamento, projeto e avaliação de tarefas e postos de trabalho, ambientes, sistemas e produtos, contribuindo majoritariamente na compatibilização dos postos de trabalho com as 
necessidades dos indivíduos direcionados. Tem-se assim o objetivo de melhorar e aumentar a qualidade de vida do indivíduo envolvido, mediante modificação de sistemas e adequação de atividades às peculiaridades das pessoas, entregando as mesmas conforto e segurança no desempenho de suas tarefas.

A partir de avaliações ergonômicas realizadas nos postos de trabalho é possível traçar um plano de intervenção que aborde as necessidades físicas, cognitivas e organizacionais envolvidas no ambiente laboral. Para estas necessidades são elaborados critérios de execução, dentro desses podem ser planejadas ações que supram os critérios e facilitem o labor. O Método de Análise Ergonômica do Trabalho (AET) propõe uma avaliação que consiste no diagnóstico real e situacional da atividade laboral e dimensiona características físicas e mentais de acordo com cada tarefa realizada. Permite, após sua aplicação, delimitação concreta dos fatores de risco e eficácia em um dado local, facilitando o conforto e segurança no trabalho (VAZ, 2015).

De Paula (2016) acrescenta que os fatores ambientais e processuais do trabalho podem ser avaliados e definidos como objetivo principal de intervenção, podendo relacionar-se com conceitos de anatomia, antropometria, fisiologia e biomecânica. As estratégias definidas para cada posto de trabalho dependem da análise ergonômica realizada, podendo ter como ações: educação em saúde, capacitação de profissionais de segurança de trabalho, programa de ginástica laboral, promoção e prevenção à saúde no ambiente de trabalho.

Um Programa de Promoção a Saúde do Trabalhador (PPST) encontra-se dentro de uma demanda de políticas e atividades que têm sido utilizadas no ambiente ocupacional objetivando melhorar a qualidade de vida do trabalhador, a partir do ambiente de trabalho, respeitando as características da empresa, do colaborador (empregado) e de todo contexto em que este se encontra (SILVA, 2014). Essa demanda pode ser composta por palestras sobre qualidade de vida relacionada à saúde e ao trabalho e é definida principalmente por meio da aplicação de questionários, avaliações e coleta de dados antropométricos.

A ginástica laboral, programas esportivos, eventos de cultura e lazer, intervenções ergonômicas, apoio emocional e cuidados com alimentação podem ser ações realizadas nas empresas a fim de favorecer a Gestão da Qualidade de Vida no trabalho. Dessa maneira, programas como a promoção de saúde no trabalho fomentados pela Ergonomia auxiliam na redução de fatores que levam o trabalhador ao risco ocupacional e ao afastamento da atividade laboral em decorrência da exposição ao risco (BARBOSA et al., 2014).

As ciências que estudam os processos de trabalho, fomentam revoluções no âmbito laboral. Dentro de suas atribuições, o Terapeuta Ocupacional do Trabalho, utilizando os princípios da Política Nacional da Saúde do Trabalhador, fundamentados nos conhecimentos técnicos e científicos da Ergonomia, e na Classificação Internacional de Funcionalidade (CIF), tem como competência promover ações profissionais, de alcance individual e/ou coletivo, de promoção à saúde (ações informativas de educação em saúde) e prevenção da incapacidade, na perspectiva do direito à saúde e da participação social como instrumento da recuperação da saúde ocupacional (COFFITO, 2015).

Terapeutas Ocupacionais que antes trabalhavam com esse público na perspectiva de reabilitação profissional, depois das mudanças de paradigmas do Sistema Único de Saúde (SUS), ganharam novos horizontes de atuação podendo dar cobertura nas intervenções de habilitação, reabilitação e recuperação profissional, seguindo as seguintes linhas de estruturação de práticas: reabilitação profissional; prevenção de doenças e acidentes; promoção e prevenção de saúde e promoção social. Tem-se como objetivos comuns a investigação da atividade laboral, das condições de trabalho, dos postos de serviço, da organização e relações de trabalho (SILVA et al., 2016).

Dessa maneira, o presente estudo tem como objetivo apresentar estratégias de intervenções da Terapia Ocupacional no ambiente de trabalho como recurso de Promoção de Saúde, sob a ótica da Ergonomia. 


\section{METODOLOGIA}

Trata-se de um estudo descritivo-exploratório, desenvolvido a partir de um relato de experiência em aulas práticas da disciplina Terapia Ocupacional na Saúde do Trabalhador, ocorridas no Departamento de Terapia Ocupacional da Universidade Federal de Pernambuco, em Recife-PE. Foram seis encontros que ocorreram no período de 2 a 30 de junho de 2016, sendo supervisionados pela docente e um monitor da disciplina, com atuação direta de quatro discentes na mediação de grupos focais, com temáticas referentes a prevenção de agravos e promoção à saúde do trabalhador.

Os dados foram coletados por meio de entrevista e observações, seguindo as fases do Método de Análise Ergonômica do Trabalho (AET) junto a três equipes de trabalho. Cada equipe era composta por um grupo de oito a quinze indivíduos de ambos os sexos, que desempenhavam a função de servidores de limpeza externa nas dependências do campus da Universidade Federal de Pernambuco. Participaram dos grupos oito indivíduos de cada equipe, em média, por encontro.

Segundo a Norma Regulamentadora $n^{\circ} 17$ (MTPS, 2007), a análise ergonômica do trabalho é um processo construtivo e participativo para a resolução de um problema complexo que exige o conhecimento das tarefas, da atividade desenvolvida para realizá-las e das dificuldades enfrentadas para se atingirem o desempenho e a produtividade exigidos.

Santos e Fialho (1997) relatam que a análise ergonômica do trabalho compreende três fases: análise da demanda, análise da tarefa e análise das atividades. $\mathrm{Na}$ análise da demanda define-se o problema a ser investigado com os atores envolvidos. A análise da tarefa coloca o que o trabalhador deve realizar e as condições ambientais técnicas e organizacionais. $E$ a análise das atividades traz efetivamente o que é realizado pelo trabalhador, o comportamento do homem no trabalho.

Além disso, a análise das atividades se trata da verificação das funções fisiológicas e psicológicas do indivíduo num determinado momento, pois corresponde ao comportamento real no trabalho para cumprimento das tarefas estabelecidas. Terapeutas Ocupacionais analisam as demandas de uma atividade ou ocupação para entender as estruturas específicas do corpo, as funções do corpo, habilidades de desempenho e padrões de desempenho que são necessários e determinam as demandas genéricas da atividade ou ocupação realizada pelo cliente (AOTA, 2015).

Após diagnóstico fornecido pelo Método de AET, foram definidas temáticas para serem abordadas nos grupos de intervenção, tais como: alongamentos e ginástica laboral; hábitos saudáveis: higiene pessoal e alimentação; riscos no trabalho: equipamentos de proteção individual (EPI); relações interpessoais no trabalho. Os dados coletados serviram de base para a escolha das temáticas e direcionamento das orientações nos quatro grupos realizados com os trabalhadores, os quais serão apresentados adiante.

\section{RESULTADOS / DISCUSSÃO}

Ao longo dos dois (2) primeiros grupos foram observados aspectos ergonômicos e processuais da atividade laboral dos trabalhadores, a partir da aplicação do Método de AET para estabelecimento dos principais objetivos para intervenção nos grupos focais. Dentre os aspectos observados destacaram-se: equipamentos; aspectos gerais de saúde; biomecânica aplicada a atividade laboral; limitações por aspectos físicos, dor, qualidade de vida e relacionamento extra pessoal no local de trabalho. Além das avaliações foi aplicado um questionário, elaborado pelos envolvidos na disciplina, em forma de entrevista.

A seguir serão apresentados os dados coletados, a partir da aplicação do Método de Análise Ergonômica do Trabalho: 


\section{Fase 1 - Análise da demanda}

Como demanda inicial, observou-se uma série de inadequações na atividade de trabalho dos funcionários analisados, desde o uso inapropriado das vestimentas e EPI utilizados, até posturas e movimentos inadequados (Figura 1). Outros aspectos observados mediante a entrevista inicial foi a má alimentação ao longo da jornada de trabalho, baseada prioritariamente de alimentos com alto teor calórico e pobre em nutrientes, como frituras e produtos industrializados.

Figura 1 - Etapa de entrevistas e Análises das atividades laborais.



Fonte: Autores

\section{Fase 2 - Análise Ergonômica da Tarefa}

As atividades dos trabalhadores de limpeza externa do Campus Recife da UFPE são diversas. É de responsabilidade deles realizarem: capinação, que envolve o corte e a limpeza; coleta e transporte de resíduos, lixos e entulhos; pequenos reparos em edificações; serviços de carpintaria e pintura; transporte de pequenas cargas; podagem de árvores e jardins externos; manipulação de máquinas agrícolas; entre outras atividades. Essas atividades podem tornar a jornada de trabalho longa e exaustiva, pois muitas vezes este trabalho é realizado em espaço aberto e com exposição direta ao sol.

Os trabalhadores realizam suas atividades, na maior parte do tempo em áreas abertas da Universidade, sendo assim expostos às chuvas, ao sol, aos riscos de acidentes, como serem atingidos por algum veículo, ou entrarem em contato com algum animal peçonhento. Este ambiente também é marcado pela presença de inúmeros ruídos, tanto das próprias máquinas que são utilizadas por eles, quanto dos automóveis que trafegam pelo Campus. Muitas vezes, as áreas em que estes trabalhadores são escalados para realizar alguma tarefa não são de simples acesso.

A jornada de trabalho que é requisitada para os mesmos é de 9 horas diárias e mais 1 hora para o descanso e almoço, os trabalhadores iniciam suas atividades às 7 horas da manhã e seguem até o meio dia, quando param para almoçar e descansar, alguns trabalhadores optam por passarem este tempo no alojamento, que por sua vez não oferece condições adequadas para o descanso e alimentação, por ser um espaço pequeno e que oferece diversos riscos aos trabalhadores, como exemplo, choque elétrico. Após o intervalo, retomam suas atividades e às 17 horas são liberados. 


\section{Fase 3 - Análise da Atividade}

Realizou-se análise das demandas da atividade ou ocupação para entender as estruturas específicas do corpo, as funções do corpo, habilidades de desempenho e padrões de desempenho que são necessários e determinam as demandas genéricas da atividade ou ocupação realizadas pelo cliente (AOTA, 2015). Análise pode ser observada na Tabela 1.

Tabela 1 - Análise da Atividade de acordo com AOTA, 2015.

\begin{tabular}{|c|c|c|c|c|}
\hline Atividade & \multicolumn{4}{|c|}{ Cortar a Grama } \\
\hline \multirow{18}{*}{$\begin{array}{l}\text { Funções do } \\
\text { Corpo }\end{array}$} & \multirow{6}{*}{ Funções Mentais } & \multirow{4}{*}{\multicolumn{2}{|c|}{ Funções Mentais Específicas }} & Atenção \\
\hline & & & & Percepção \\
\hline & & & & Pensamento \\
\hline & & & & $\begin{array}{l}\text { Função mental para } \\
\text { o sequenciamento de } \\
\text { movimentos } \\
\text { complexos }\end{array}$ \\
\hline & & \multirow{2}{*}{\multicolumn{2}{|c|}{ Funções Mentais Globais }} & Consciência \\
\hline & & & & Energia e disposição \\
\hline & \multirow{3}{*}{ Funções sensoriais } & Função visual & & \\
\hline & & \begin{tabular}{|l|} 
Função \\
proprioceptiva
\end{tabular} & & \\
\hline & & Função tátil & & \\
\hline & \multirow{8}{*}{$\begin{array}{c}\text { Funções } \\
\text { neuromusculo- } \\
\text { esqueléticas }\end{array}$} & \multirow{2}{*}{$\begin{array}{c}\text { Funções articulares } \\
\text { e ósseas }\end{array}$} & $\begin{array}{l}\text { Mobilidade } \\
\text { articular }\end{array}$ & \\
\hline & & & $\begin{array}{l}\text { Estabilidade } \\
\text { articular }\end{array}$ & \\
\hline & & \multirow{3}{*}{$\begin{array}{l}\text { Funções } \\
\text { musculares }\end{array}$} & Força muscular & \\
\hline & & & Tônus muscular & \\
\hline & & & $\begin{array}{l}\text { Resistência } \\
\text { muscular }\end{array}$ & \\
\hline & & \multirow{3}{*}{$\begin{array}{l}\text { Funções de } \\
\text { movimento }\end{array}$} & Reflexos motores & \\
\hline & & & \begin{tabular}{|l} 
Reações de \\
movimentos \\
involuntários
\end{tabular} & \\
\hline & & & $\begin{array}{l}\text { Controle do } \\
\text { movimento } \\
\text { voluntário }\end{array}$ & \\
\hline & $\begin{array}{l}\text { Funções do sistema } \\
\text { cardiorespiratório }\end{array}$ & $\begin{array}{c}\text { Funções e } \\
\text { sensações } \\
\text { adicionais dos } \\
\text { sistemas }\end{array}$ & & \\
\hline $\begin{array}{c}\text { Estruturas do } \\
\text { corpo }\end{array}$ & \begin{tabular}{|l|} 
Estrutura do sistema \\
nervoso \\
Estruturas dos \\
sistemas \\
cardiovascular e \\
respiratório \\
\end{tabular} & & & \\
\hline
\end{tabular}




\begin{tabular}{|c|c|c|}
\hline & \begin{tabular}{|l} 
Estruturas \\
relacionadas ao \\
movimento
\end{tabular} & \\
\hline \multirow{21}{*}{$\begin{array}{c}\text { Habilidades } \\
\text { de } \\
\text { Desempenho }\end{array}$} & \multirow{12}{*}{ Habilidades motoras } & Estabilidade \\
\hline & & Posicionamento \\
\hline & & Alcance \\
\hline & & Inclinar \\
\hline & & Preensão \\
\hline & & Manipulação \\
\hline & & Coordenação \\
\hline & & Mover \\
\hline & & Sustentação \\
\hline & & Transporte \\
\hline & & $\begin{array}{l}\text { Calibração } \\
\text { Refinamento }\end{array}$ \\
\hline & & Resistência \\
\hline & \multirow{8}{*}{$\begin{array}{l}\text { Habilidades } \\
\text { processuais }\end{array}$} & Ritmo \\
\hline & & Atenção \\
\hline & & Aplicabilidade \\
\hline & & Manipulação \\
\hline & & Sequenciamento \\
\hline & & Organização \\
\hline & & Restabelecer \\
\hline & & Ajustamento \\
\hline & $\begin{array}{l}\text { Habilidades de } \\
\text { interação social }\end{array}$ & Prestar Atenção \\
\hline
\end{tabular}

Fonte: Autores

\section{Fases 4 e 5 - Diagnose e Recomendações ergonômicas}

Todo e qualquer posto de trabalho deve atender as necessidades psicofisiológicas dos trabalhadores, conforme a NR-17 (MTPS, 2007). Essa necessidade se dá ao fato de que o indivíduo que possui os fatores de riscos (biológico, químico, físico, ergonômico e de acidente) minimizados por adequação do trabalho as suas peculiaridades, desempenha melhor as suas funções.

Segundo conhecimentos pertinentes a ergonomia física, dados antropométricos, fisiológicos, anatômicos e biomecânicos (DE PAULA, 2016), pode-se destacar que um importante aspecto de início para intervenções foi a má postura ao longo da atividade desempenhada. Esse fator é decorrente da precária instrução dos funcionários para a saúde do corpo, tendo em vista a frequente queixa de dor e vício postural. Segundo Da Silva (2006), a manutenção de posturas inadequadas e desconfortos provocados pela repetição de atividades podem causar adoecimentos ocupacionais.

Outra característica analisada no posto de trabalho foi o uso inadequado de EPI, bem como, a falta de manutenção em alguns. Os EPI são importantes recursos para estabelecimentos de uma atividade laboral satisfatória e com poucos riscos de acidente. Segundo a NR-6, cabe ao trabalhador utilizar todos os EPIs solicitados para a atividade e manter a sua conservação e higienização (MTPS, 2001) 
Torna-se importante destacar ainda que a alimentação é uma rica fonte de saúde e como tal precisa ser mantida em constante harmonia com as necessidades do cotidiano. Segundo De Matos (2000), estratégias que objetivam a melhora na qualidade nutricional permitem que o indivíduo tenha a sua saúde ampliada devido a melhora na qualidade de vida e nas condições nutricionais. Permite-se também um aumento na resistência e capacidade física, além de reduzir os riscos de acidentes de trabalho.

Tendo em vista as análises realizadas, é válido ressaltar a necessidade de implantação de rotinas de práticas com o corpo, a Ginástica Laboral, como exemplo, em ao menos um horário dentro da rotina de trabalho. O comprometimento grupal com uma prática de fortalecimento de vínculo e autogestão podem ser utilizadas como estratégia de engajamento do grupo em atividades extras (SERRA, 2015).

\section{Intervenção da Terapia Ocupacional}

A partir do Método de AET, observou-se: ausência de práticas corporais, como alongamento, no cotidiano de trabalho; constante relato de dor física no final da jornada de trabalho; presença de fatores predisponentes para doenças ocupacionais, como a repetição excessiva de movimentos ao longo da jornada de trabalho; dados antropométricos evidenciando o sobrepeso e subnutrição; má alimentação durante as refeições e intervalos; e, déficits no autocuidado. Outros dados obtidos nas avaliações que podem ser ressaltados foram: limitações físicas no ambiente de trabalho; limitações cognitivas e emocionais; alterações na capacidade funcional.

Desta forma, foram elencadas algumas temáticas abordadas nos grupos de intervenção, cujos objetivos e atividades estão descritos na tabela 2 e ilustrados nas figuras 2 e 3.

Tabela 2. Temáticas dos grupos de Intervenção em Terapia Ocupacional.

\begin{tabular}{|c|c|c|c|}
\hline TEMÁTICA & OBJETIVO & ABORDAGEM & ATIVIDADES \\
\hline \multirow{4}{*}{$\begin{array}{l}\text { Alongamentos } \\
\text { e ginástica } \\
\text { laboral }\end{array}$} & Reduzir a dor & $\begin{array}{l}\text { Atividade física de } \\
\text { alongamento }\end{array}$ & $\begin{array}{l}\text { Apresentação de técnicas de } \\
\text { alongamento }\end{array}$ \\
\hline & $\begin{array}{l}\text { Melhorar a } \\
\text { qualidade de vida }\end{array}$ & $\begin{array}{l}\text { Prática corporal } \\
\text { relacionada ao } \\
\text { trabalho }\end{array}$ & $\begin{array}{l}\text { Exercícios de } \mathrm{GL}^{*} \text { relacionadas a } \\
\text { função no trabalho }\end{array}$ \\
\hline & $\begin{array}{l}\text { Buscar a } \\
\text { promoção de } \\
\text { saúde }\end{array}$ & \multirow[t]{2}{*}{$\begin{array}{l}\text { Atividade física de } \\
\text { alongamento e } \\
\text { consciência corporal }\end{array}$} & \multirow[t]{2}{*}{$\begin{array}{l}\text { Atividade de relaxamento e } \\
\text { respiração }\end{array}$} \\
\hline & $\begin{array}{l}\text { Proporcionar } \\
\text { uma prática de } \\
\text { saúde física }\end{array}$ & & \\
\hline TEMÁTICA & OBJETIVO & ABORDAGEM & ATIVIDADES \\
\hline \multirow[t]{2}{*}{$\begin{array}{l}\text { Hábitos } \\
\text { saudáveis: } \\
\text { higiene } \\
\text { pessoal e } \\
\text { alimentação }\end{array}$} & $\begin{array}{l}\text { Realizar } \\
\text { atividades de } \\
\text { Educação em } \\
\text { Saúde }\end{array}$ & $\begin{array}{l}\text { Atividades lúdicas e } \\
\text { expressivas } \\
\text { abordando a temática }\end{array}$ & $\begin{array}{l}\text { "Quantas calorias tem" (Atividade de } \\
\text { percepção calórica dos alimentos) }\end{array}$ \\
\hline & $\begin{array}{l}\text { Estimular hábitos } \\
\text { saudáveis }\end{array}$ & $\begin{array}{l}\text { Exposição de } \\
\text { imagens explicativas }\end{array}$ & \\
\hline
\end{tabular}




\begin{tabular}{|c|c|c|c|}
\hline & $\begin{array}{l}\text { Promover a } \\
\text { substituição de } \\
\text { alimentos }\end{array}$ & \multirow[t]{2}{*}{$\begin{array}{l}\text { e construção do } \\
\text { conhecimento }\end{array}$} & \multirow[t]{2}{*}{$\begin{array}{l}\text { Perguntas "Verdadeiro ou Falso" } \\
\text { (Alimentos saudáveis e valor } \\
\text { energético) }\end{array}$} \\
\hline & $\begin{array}{l}\text { Orientar quanto a } \\
\text { produtos }\end{array}$ & & \\
\hline TEMÁTICA & OBJETIVO & ABORDAGEM & ATIVIDADES \\
\hline \multirow[t]{3}{*}{$\begin{array}{l}\text { Riscos no } \\
\text { Trabalho: } \\
\text { Equipamentos } \\
\text { de Proteção } \\
\text { Individual }\end{array}$} & $\begin{array}{l}\text { Explorar de } \\
\text { maneira } \\
\text { expositiva os } \\
\text { riscos no } \\
\text { ambiente laboral }\end{array}$ & Educação em Saúde & $\begin{array}{l}\text { Demonstrações dos Riscos no } \\
\text { trabalho. }\end{array}$ \\
\hline & $\begin{array}{l}\text { Classificar e } \\
\text { identificar as } \\
\text { melhores formas } \\
\text { de prevenção de } \\
\text { riscos }\end{array}$ & $\begin{array}{l}\text { Exposição de } \\
\text { conteúdo }\end{array}$ & $\begin{array}{l}\text { Quiz com perguntas e respostas } \\
\text { sobre os equipamentos de proteção } \\
\text { individual. }\end{array}$ \\
\hline & $\begin{array}{l}\text { Trabalhar a } \\
\text { importância do } \\
\text { uso, limpeza e } \\
\text { armazenamento } \\
\text { de EPls }\end{array}$ & $\begin{array}{l}\text { Discussão de } \\
\text { solução-problema }\end{array}$ & $\begin{array}{l}\text { Como identificar os riscos no } \\
\text { trabalho. }\end{array}$ \\
\hline TEMÁTICA & OBJETIVO & ABORDAGEM & ATIVIDADES \\
\hline \multirow[t]{3}{*}{$\begin{array}{l}\text { Relações } \\
\text { interpessoais } \\
\text { no Trabalho }\end{array}$} & $\begin{array}{l}\text { Melhorar a } \\
\text { qualidade de } \\
\text { vida, decorrente } \\
\text { de uma boa } \\
\text { relação entre as } \\
\text { equipes de } \\
\text { trabalho }\end{array}$ & \multirow[t]{3}{*}{$\begin{array}{l}\text { Atividades lúdicas e } \\
\text { expressivas } \\
\text { abordando a temática }\end{array}$} & \multirow[t]{3}{*}{$\begin{array}{l}\text { Atividades lúdicas e expressivas, abo } \\
\text { rdando a temática. }\end{array}$} \\
\hline & $\begin{array}{l}\text { Trabalhar } \\
\text { confiança, auto } \\
\text { percepção e } \\
\text { percepção grupal }\end{array}$ & & \\
\hline & $\begin{array}{l}\text { Estimular o } \\
\text { trabalho em } \\
\text { equipe }\end{array}$ & & \\
\hline
\end{tabular}

*GL = Ginástica Laboral

Fonte: Autores 
Figura 2 - Exposição dos EPls e riscos no trabalho

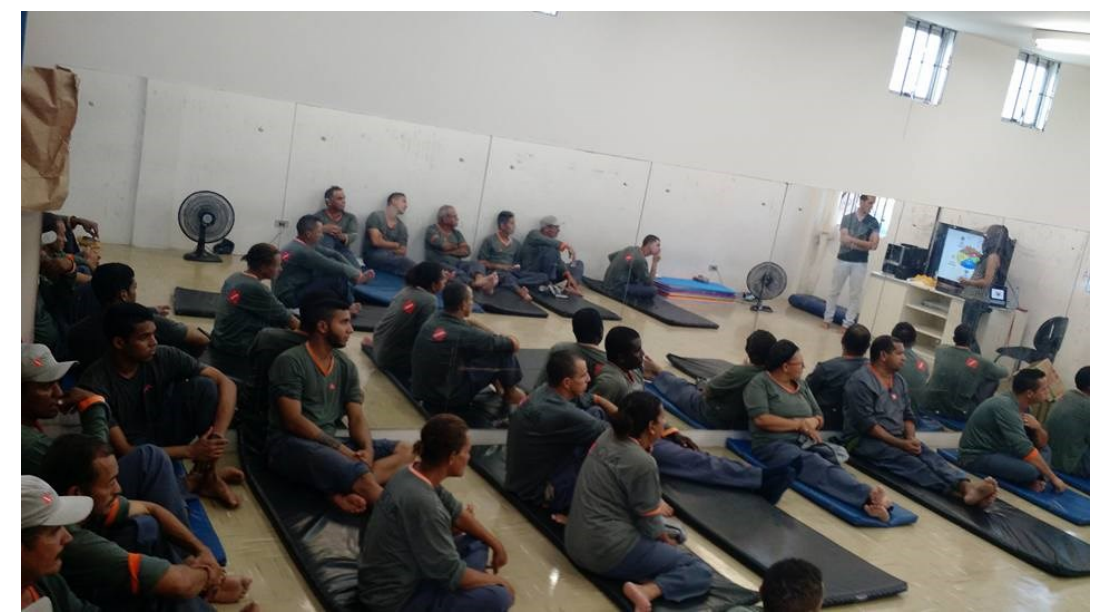

Fonte: Autores

Figura 3 - Ginástica laboral



Fonte: Autores

\section{CONCLUSÃO}

De acordo com o exposto, foi possível perceber que a partir de uma ação voltada para a promoção de saúde no ambiente de trabalho, realizada mediante prévia análise ergonômica, com respeito às características da empresa e do corpo de funcionários, há ganhos para a relação homem-trabalho, favorecendo-se a aprendizagem e a adoção de um estilo de vida saudável no trabalho, com qualidade e eficácia.

Ao utilizar o Programa de Promoção à Saúde do Trabalhador, como estratégia de intervenção, o terapeuta ocupacional tem o objetivo de melhorar a qualidade de vida do trabalhador a partir da melhoria do ambiente de trabalho de forma ampla, física e organizacional.

A Ginástica Laboral entra nessa discussão como sendo um recurso de promoção de saúde que apresenta ênfase na prevenção de agravos e compensação à carga de trabalho. Contribui significativamente por preparar a musculatura para o desempenho de funções especificas, prevenindo lesões relacionadas a mesma, podendo ainda ser utilizada com recurso de fortalecimento de vínculo e facilitadora no processo de resolução de conflitos, como foi possível observar nos grupos. 


\section{REFERÊNCIAS BIBLIOGRÁFICAS}

AOTA - ASSOCIAÇÃO AMERICANA DE TERAPIA OCUPACIONAL et al. Estrutura da prática da Terapia Ocupacional: domínio \& processo-traduzida. Revista de Terapia Ocupacional da Universidade de São Paulo, v. 26, n. esp, p. 1-49, 2015.

BARBOSA, P. H. et al. Doenças osteomusculares relacionadas ao trabalho e à ginástica laboral como estratégia de enfrentamento. Archives of Health Investigation, v. 3, n. 5, 2014.

COFFITO. Conselho Federal de Fisioterapia e Terapia Ocupacional. Resolução - COFFITO $\mathbf{N}^{\circ}$ 459/2015. Dispõe sobre as competências do terapeuta ocupacional na Saúde do Trabalhador, atuando em programas de estratégias inclusivas de prevenção, proteção e recuperação da saúde. Brasília, 20 nov. 2015. Disponível em: $<$ <ttp://www.coffito.org.br/site/index.php/home/resolucoes-coffito/966-resolucao-n-459-de-20de-novembro-de-2015-dispoe-sobre-as-competencias-do-terapeuta-ocupacional-na-saudedo-trabalhador-atuando-em-programas-de-estrategias-inclusivas-de-prevencao-protecao-erecuperacao-da-saude.html>

DA SILVA, J. A. A. B.; TARANTO, I. C.; PIASECKI, F. Ginástica Laboral: alongamento $x$ flexionamento. SaBios-Revista de Saúde e Biologia, v. 1, n. 2, 2006.

DE OLIVEIRA, J. R. G. A importância da ginástica laboral na prevenção de doenças ocupacionais. Revista de Educação Física, v. 139, p. 40-49, 2007.

DE PAULA, A.; HAIDUKE, I. F.; MARQUES, I. A. A. Ergonomia e Gestão: complementaridade para a redução dos afastamentos e do stress, visando melhoria da qualidade de vida do trabalhador. Revista Conbrad, v. 1, n. 1, p. 121-136, 2016.

JUNQUEIRA, T. Trabalho, Saúde e Terapia Ocupacional: uma abordagem sistêmica. In: Congresso Brasileiro de Sistemas, 4., 2008. São Paulo. Anais... São Paulo, 2008. p. 1-16.

KANTORSKI, L. P. As transformações no mundo do trabalho e a questão da saúde: algumas reflexões preliminares. Revista Latino-Americana de Enfermagem, v. 5, n. 2, p. 5-15, 1997.

LANCMAN, S. Psicodinâmica do Trabalho. In: CAVALCANTI, A.; GALVÃO, C. Terapia Ocupacional: Fundamentação e Prática. Cap. 28. p. 271-277.

MORETTI, S.; TREICHEL, A. Qualidade de Vida no Trabalho e auto-Realização Humana. Revista Leonardo pós-Órgão de Divulgação Científica e Cultural do ICPG, Blumenau, 1,3, p.73-80, 2003.

MTPS - Ministério do Trabalho e da Previdência Social. NR 17 - Ergonomia. 2007. Disponível em: <http://www.mtps.gov.br/images/Documentos/SST/NR/NR17.pdf>

NR 6 - Equipamentos de Proteção Individual. 2001. Disponível em:

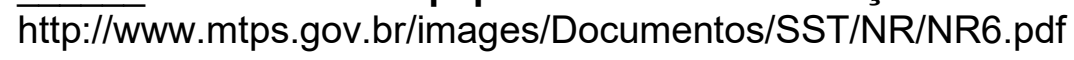

PAIVA FILHO, E. M.; CARVALHO, N.; TEIXEIRA, K. M. A importância da Ginástica Laboral na prevenção de doenças ocupacionais. Anais da Semana de Licenciatura, v. 1, p. 155-165, 2010.

SANTOS, N.; FIALHO, F. Manual de análise ergonômica do trabalho. Curitiba: Gênesis, v. 2, p. 316, 1997. 
SARAIVA, F. F. et al. Análise Ergonômica Do Trabalho: Um Estudo De Casos Múltiplos na Cidade Do Rio De Janeiro. In: XIV Mostra de Iniciação Científica, Pós-graduação, Pesquisa e Extensão. 2014.

SERRA, M. V. G. B.; PIMENTA, L. C.; QUEMELO, P. R. V. EFEITOS DA GINÁSTICA LABORAL NA SAÚDE DO TRABALHADOR: UMA REVISÃO DA LITERATURA. Revista Pesquisa em Fisioterapia, v. 4, n. 3, 2015.

SILVA, F. M. N.; VENDRÚSCULO-FANGEL, L. M.; DA SILVA RODRIGUES, D. A Terapia Ocupacional e a saúde do trabalhador: panorama de produção bibliográfica/Occupational Therapy and workers' health: an overview of the bibliographic production. Cadernos de Terapia Ocupacional da UFSCar, v. 24, n. 2, 2016.

SILVA, R. et al. Influência de Informações de Saúde no Estilo de Vida de Participantes de Ginástica Laboral. Revista Brasileira de Promoção a Saúde, Fortaleza, v. 27, n. 3, p.406412, jul./set. 2014.

VAZ, R. S. C. A atuação da terapia ocupacional no Centro de Referência em Saúde do Trabalhador: uma revisão bibliográfica (2007 a 2014). Disponível em: <http://bdm.unb.br/handle/10483/10336> Acesso em: 15 jul. 2016.

VERGARA, Lizandra Garcia Lupi et al. Análise Ergonômica da atividade de Jardinagem e Paisagismo. Revista Eletrônica Produção em Foco, v. 2, n. 1, 2012. 